\title{
On the velocity of planar trajectories
}

\author{
Zvi Artstein and Ido Bright
}

\begin{abstract}
Consider two trajectories each forming a Jordan curve with a finite length in the two dimensional plane. We show that the integral of the velocity of the portion of one curve that is included in the inside of the second curve, is bounded by half the length of the latter curve. We point out an application of the result to averaging.
\end{abstract}

Mathematics Subject Classification (2010). Primary 34C05; Secondary 34C25, 34C29.

Keywords. Planar periodic orbits, Jordan curves.

\section{The main result}

Consider a Jordan curve, say $J$, with a finite length $L$ in the two-dimensional plane $R^{2}$. It divides the plane into two regions, the inside of $J$, that we denote by $\operatorname{In}(J)$, and the outside of $J$, that we denote by $\operatorname{Out}(J)$. To avoid ambiguity we agree not to include in $\operatorname{In}(J)$ the curve itself; thus the closure of $\operatorname{In}(J)$ is $\operatorname{In}(J)$ union $J$; likewise with respect to the outside of $J$.

Consider another Jordan curve in the plane, given by $x(t):[0, T] \rightarrow R^{2}$, namely $x(0)=x(T)$ and otherwise $x\left(t_{1}\right) \neq x\left(t_{2}\right)$ if $t_{1} \neq t_{2}$. Suppose $x(\cdot)$ is absolutely continuous and let $u(t)$ denote its almost everywhere derivative. Denote by $|\cdot|$ the Euclidean norm on $R^{2}$. Our main result is as follows.

Theorem A. Let $U$ denote the subset of $[0, T]$ for which $x(t) \in \operatorname{In}(J) \cup J$. Then

$$
\left|\int_{U} u(t) d t\right| \leq \frac{L}{2}
$$

Remark 1.1. Since $\int_{0}^{T} u(t) d t=0$ and since the roles of inside and outside are interchangeable, it follows that (1.1) holds also when $U$ is the subset of $[0, T]$ where $x(t)$ is in $\operatorname{Out}(J)$, hence also when $U$ is the subset of $[0, T]$ where

This paper is dedicated to Arrigo Cellina on the occasion of his 70th birthday.

Z. Artstein is the Incumbent of the Hettie H. Heineman Professorial Chair in Mathematics. Research supported by a grant from the Israel Science Foundation. 
$x(t)$ belongs to $\operatorname{In}(J)$ and, likewise, when $U$ is the subset of $[0, T]$ where $x(t)$ belongs to $\operatorname{Out}(J) \cup J$.

The result is an extension of an observation that served us (in [1]) in establishing a Poincaré-Bendixson type result for infinite horizon optimal control problems, namely, verifying that if the state space is two-dimensional, it suffices to consider periodic solutions. We allude to this application in the closing section. In the next two sections we verify the result in two special cases, this in preparation to the completion of the proof of the theorem in Sect. 4, for which we establish some useful observations concerning approximations of Jordan curves. In the closing section we provide some examples and comments, showing, in particular, that the estimates are sharp, but can be improved when $J$ is convex.

\section{The case of a finite number of intersections}

In the sequel, and without loss of generality, we assume that $J$ is parameterized as $y(s):[0, L] \rightarrow R^{2}$, where $y(\cdot)$ is absolutely continuous, $y(0)=y(L)$ and otherwise $y\left(s_{1}\right) \neq y\left(s_{2}\right)$ if $s_{1} \neq s_{2}$, and such that $\left|\frac{d y}{d s}\right|=1$ for every $s$. (Here and in the sequel we reserve the variable $s$ to indicate the parameterization of $J$.) This is the standard arc-length parameterization; it can be constructed by identifying one point on $J$ as $y(0)$, prescribe an orientation on $J$ and then define $y(s)$ to be the point on $J$ with distance from $y(0)$, on $J$ in the positive direction, of magnitude $s$. In particular, intervals on $J$ correspond to intervals on $[0, L]$ when 0 is identified with $L$. We denote then $\frac{d y}{d s}(s)=v(s)$.

Before stating and verifying our result in a special case as follows, we display a claim that holds in general and, in fact, holds for periodic orbits in any dimension.

Lemma 2.1. Let $V$ be a Borel measurable subset of $[0, L]$. Then

$$
\left|\int_{V} v(s) d s\right| \leq \frac{L}{2} .
$$

Proof. Since $\int_{0}^{L} v(s) d s=0$ it follows that if $\left|\int_{V} v(s) d s\right|>\frac{L}{2}$ then the norm of the integral on the complement of $V$ in $[0, L]$ is also greater than $\frac{L}{2}$. This would imply that $\int_{0}^{L}|v(s)| d s>L$, in contradiction with $\int_{0}^{L}|v(s)| d s$ being the length of $J$, namely equal to $L$. This completes the proof.

Here is the special case announced in the title of the section.

Proposition 2.2. Suppose that the curve $x(\cdot)$ intersects $J$ in only a finite number of points. Then (1.1) holds.

Proof. A key observation is that $\int_{0}^{T} u(t) d t=0$, which follows from the periodicity. Hence the cases where $U=[0, T]$ or $U=\emptyset$ are trivial, and likewise (since the outside can play the role of inside) when $x(t)$ is always in $\operatorname{Out}(J) \cup J$. Hence we have to consider only the case where the trajectory $x(\cdot)$ intersects both $\operatorname{In}(J)$ and $\operatorname{Out}(J)$ and, in particular, intersects $J$ itself. 
Among the finite number of points of intersection there are those where $x(\cdot)$ stays on the same side of $J$ on short intervals before and after the intersection, and those for which the trajectory crosses the Jordan curve at the intersection, either from $\operatorname{In}(J)$ to $\operatorname{Out}(J)$ or from $\operatorname{Out}(J)$ to $\operatorname{In}(J)$. We consider now only the points of crossing. Let $z_{1}$ be one of these points. We choose an orientation on $J$, say clockwise, and denote by $z_{2}, z_{3}, \ldots, z_{n}$ the other points at which $x(\cdot)$ crosses $J$, in the order they appear on $J$ according to the chosen orientation (which may not necessarily be the order derived from $[0, T]$ ). We also identify $z_{1}=z_{n+1}$. We shall need the following observation.

Observation. If $z_{i}$ is a point where $x(\cdot)$ crosses $J$ from inside to outside (or from outside to inside) then $z_{i+1}$ is a point where $x(\cdot)$ crosses $J$ from outside to inside (respectively, from inside to outside). In particular, $n$ is even.

To verify the observation we use the two-dimensional topology. Let $J_{1}$ be the curve that consists of the range of $x(\cdot)$ starting at the time $t_{i}$ where $x\left(t_{i}\right)=z_{i}$, until the time $t_{i+1}$ where $x\left(t_{i+1}\right)=z_{i+1}$. Consider the concatenation of $J_{1}$ with the segment of $J$ that joins $z_{i}$ and $z_{i+1}$. If $x(\cdot)$ crosses $J$ from inside to outside both at $z_{i}$ and at $z_{i+1}$, then in order to reach $z_{i}$ again, which it should due to periodicity, $x(\cdot)$ will have to cross the concatenated curve. This is not allowed since the curve induced by $x(\cdot)$ is Jordan and since there are no crossing points on $J$ between $z_{i}$ and $z_{i+1}$. The contradiction verifies the observation.

We continue with the proof of the proposition. We construct now a finite number of periodic trajectories $\theta_{1}, \ldots, \theta_{m}$, each composed of portions of $x(\cdot)$ in $\operatorname{In}(J)$ and portions of $J$ itself, such that each $\theta_{i}$ intersects itself in at most a finite number of times and any two distinct $\theta_{i}$ intersect each other in at most a finite number of points (in fact, it is possible to show that they do not intersect but we shall not need it in the proof), and the union of $\theta_{i}, i=1, \ldots, m$, contains the range of $x(\cdot)$ within $\operatorname{In}(J)$ and a portion of $J$ (but may not contain the entire curve $J$ ).

Suppose that $z_{1}$ is a point of crossing from $\operatorname{In}(J)$ to Out $(J)$. The construction of $\theta_{1}$ starts at $z_{1}$ in the clockwise direction on $J$ until $z_{2}$ is reached. By the displayed Observation, the point $z_{2}$ is a point of crossing from $\operatorname{Out}(J)$ to $\operatorname{In}(J)$. Now, $\theta_{1}$ continues by following $x(\cdot)$ in $\operatorname{In}(J)$ until a point of crossing from $\operatorname{In}(J)$ to Out $(J)$ is reached; if this point is $z_{1}$ then $\theta_{1}$ has been completed. If it is a different point, say $z_{j}$, we switch at $z_{j}$ to a portion of $J$ in the clockwise direction, until we reach $z_{j+1}$ which, by the previous Observation, is a point of crossing from $\operatorname{Out}(J)$ to $\operatorname{In}(J)$. The latter point cannot be $z_{2}$ as $z_{2}$ has been reached by the clockwise arc starting at $z_{1}$. We continue from $z_{j+1}$ with $x(\cdot)$ inside $J$ until a point of crossing outside is reached again; if this point is $z_{1}$ then $\theta_{1}$ has been completed; otherwise we continue in the same manner as before. This process must end with reaching the original $z_{1}$; indeed, since $x(\cdot)$ forms a Jordan curve the first time the process reaches a point that is already in the constructed trajectory this point must be $z_{1}$. The trajectory $\theta_{1}$ may intersect itself but only on the portions of $J$ contained in it, this since $x(\cdot)$ forms a Jordan curve; hence the self intersection of $\theta_{1}$ may occur only at a finite number of points. 
If the periodic trajectory constructed in the first stage has consumed all the crossing points we are done, in one step. Otherwise, there are crossing points not on the constructed trajectory. Consider a point, say $z_{i}$, that is on $\theta_{1}$ but such that $z_{i+1}$ is not in $\theta_{1}$. Then $z_{i}$ is a point of crossing inside $J$, otherwise the arc joining $z_{i}$ and $z_{i+1}$ would have been in $\theta_{1}$. By the Observation, the point $z_{i+1}$ crossing from $\operatorname{In}(J)$ to $\operatorname{Out}(J)$. We start now $\theta_{2}$ with $z_{i+1}$ following the process described in the construction of $\theta_{1}$. (It is not difficult to argue that all the crossing points on $\theta_{2}$ are on a segment of $J$ with interior that does not contain crossing points of $\theta_{1}$, but we do not need it in the proof.) The curve $\theta_{2}$ does not cross $\theta_{1}$ in the inside of $J$, and if it crosses it on $J$ (it is not difficult to show that this cannot happen, but we do not need it in the proof) it is only in a finite number of points. Therefore, the construction of $\theta_{2}$ ends when $z_{i+1}$ is reached again, where $\theta_{2}$ is completed.

If needed, we continue successively. After a finite number of iterations the construction of $\theta_{1}, \ldots, \theta_{m}$ is complete with the desired properties being satisfied.

With the construction of the curves $\theta_{1}, \ldots, \theta_{m}$ the completion of the proof of the proposition is simple. Indeed, the periodic curves we constructed are the images of intervals in $[0, T]$ whose union is $U$, and intervals in $[0, L]$, whose union is, say, $V$. The periodicity of the curves $\theta_{i}$ implies that the sum of the integrals of $u(\cdot)$ and $v(\cdot)$ on, respectively, $U$ and $V$, results with the vector 0 . In view of Lemma 2.1 the integral of $v(\cdot)$ over $V$ results in a vector of norm less than or equal to $\frac{L}{2}$. Hence the norm of the integral of $u(\cdot)$ on $U$ is also less than or equal to $\frac{L}{2}$ which is what is claimed in (1.1). This completes the proof of Proposition 2.2.

\section{The smooth and the convex cases}

We now state and verify the result under an additional condition as follows. Let $D_{\varepsilon}$ denote the $\varepsilon$-neighborhood of $\operatorname{In}(J) \cup J$. Denote the boundary of $D_{\varepsilon}$ by $J_{\varepsilon}$.

Proposition 3.1. Assume that for $\varepsilon$ small enough the curves $J_{\varepsilon}$ are Jordan curves. Then (1.1) holds.

Proof. We shall employ the following observation.

Auxiliary Proposition. For a set of $\varepsilon$ of full Lebesgue measure around 0 the range of $x(\cdot)$ intersects $J_{\varepsilon}$ in only a finite number of points.

To verify the auxiliary proposition consider a re-parameterization of $x(\cdot)$, say $x_{1}(\tau):\left[0, T_{1}\right] \rightarrow R^{2}$, such that $x_{1}(\tau)$ is Lipschitz in the variable $\tau$. Such a parameterization clearly exists (compare with the first paragraph of the previous section). Since the new parameterization does not change the number of intersections with each $J_{\varepsilon}$, it is enough to verify the claim with respect to this new parameterization. Consider now the mapping $r(\tau)=\varepsilon$ when $x_{1}(\tau) \in J_{\varepsilon}$. It is well defined and its range includes an open interval around 0. This mapping is Lipschitz. Now the result follows from the extension of Sard's theorem for Lipschitz maps, see Wells [7]. 
With the previous auxiliary proposition the conclusion of the proof of the proposition is easy. First, recall Steiner's theorem (see, e.g., Guggenheimer [5, page 81]) which implies that the length $L_{\varepsilon}$ of $J_{\varepsilon}$ satisfies

$$
L_{\varepsilon} \leq L+2 \pi \varepsilon
$$

Denote by $U_{\varepsilon}$ the set of points on $[0, T]$ where $x(t)$ is inside or on $J_{\varepsilon}$. By Proposition 2.2 for a set of $\varepsilon$ of full measure around 0 , the estimate (1.1) holds when we replace $U$ by $U_{\varepsilon}$. The intersection of the closed sets $U_{\varepsilon}$ for $\varepsilon$ positive is $U$. Hence (1.1) holds for the original $U$ and Proposition 3.1 is verified.

Corollary 3.2. Suppose that $J$ is either twice continuously differentiable or that $\operatorname{In}(J)$ is a convex set. Then (1.1) holds.

Proof. In both cases it is easy to see that the condition of Proposition 3.1 is met.

\section{The general case}

In this section we prove Theorem A. We adopt the setting displayed at the beginning of Sect. 2. Our first aim is a construction of an appropriate Jordan curve approximation, as follows.

Proposition 4.1. For every $\eta>0$ there exists a Jordan curve $J_{\eta, 0}$, with distance $\eta$ from $J$, with length $L_{\eta, 0}$ less than or equal to $L+2 \pi \eta$, such that the intersection of the insides of $J_{\eta, 0}$ is $\operatorname{In}(J) \cup J$.

Proof. For $\eta>0$ let $D_{\eta}$ be the closed $\eta$-neighborhood of $\operatorname{In}(J) \cup J$, namely, the set of points in $R^{2}$ whose Euclidean distance from $\operatorname{In}(J) \cup J$ is less than or equal to $\eta$. Then the interior of $D_{\eta}$ is arc-wise connected. Indeed, the interior of $D_{\eta}$ is the union of $\operatorname{In}(J)$ with the open $\eta$-neighborhood of the Jordan curve $J$.

Now consider the boundary $\partial D_{\eta}$ of $D_{\eta}$. By Steiner's theorem, the boundary $\partial D_{\eta}$, also called the parallel curve, is closed and has length, say $L_{\eta}$, satisfying

$$
L_{\eta} \leq L+2 \pi \eta
$$

See Guggenheimer [5, page 81].

The boundary curve $\partial D_{\eta}$ may not be Jordan (if it is, the proof is finished). Consider the complement of $D_{\eta}$. It is an open set. It may have many connected components.

Claim 1. In the one-point compactification of $R^{2}$ (namely considering it on the two dimensional sphere in three space) any component of the complement of $D_{\eta}$ is simply connected.

To verify the claim notice that otherwise the component would include a Jordan curve with points of $D_{\eta}$ inside and outside it, in contradiction to $D_{\eta}$ being arc-wise connected.

Consider now one component, say $O_{\eta}$ of the complement of $D_{\eta}$, and denote its boundary by $J_{\eta}$. 
Claim 2. The boundary $J_{\eta}$ of a component $O_{\eta}$ of the complement of $D_{\eta}$ is a Jordan curve with length less than or equal to $L+2 \pi \eta$.

Since $J_{\eta}$ is part of $\partial D_{\eta}$, each point on the curve $J_{\eta}$ is a boundary point of the interior of $D_{\eta}$. If the complement of $J_{\eta}$ has more than two connected components, then two distinct such components will contain points in the interior of $D_{\eta}$. This contradicts Claim 1 ; indeed, any arc that connects two points in distinct components must intersect $J_{\eta}$, hence intersects $\partial D_{\eta}$ and cannot be included in the interior of $D_{\eta}$. This proves that $J_{\eta}$ is Jordan. Being also a closed subset of $\partial D_{\eta}$ implies, in view of (4.1), the estimate on its length.

The previous two claims apply to any component of the complement of $D_{\eta}$. To prove the proposition we choose one of these components. To this end we identify a point $x_{0}$ in $\operatorname{Out}(J)$. This point will be fixed for every small enough $\eta$. Suppose that $\eta$ is smaller than the distance between $x_{0}$ and $J$. Among the open components of the complement of $D_{\eta}$ we choose the one that contains $x_{0}$, say $O_{\eta, 0}$. Its boundary is the Jordan curve $J_{\eta, 0}$. We call $O_{\eta, 0}$ the outside of $J_{\eta, 0}$. We claim that the family $J_{\eta, 0}$ satisfies the requirements of the proposition.

Being part of $\partial D_{\eta}$ the requirements on the distance from $J$ and the length of $J_{\eta, 0}$ (see Claim 2) are met. It remains to prove the statement concerning the intersection of the insides of $J_{\eta, 0}$.

Claim 3. For every point $x$ in $\operatorname{Out}(J)$, if $\eta$ is small enough, then $x$ belongs to the outside of $J_{\eta, 0}$.

To verify the claim we employ the fact that $J$ is Jordan, hence we can connect $x_{0}$ and $x$ with a closed curve, say $C$, within Out $(J)$. Let $\delta$ be the least distance between points of $C$ and of $J$, namely, for each $y \in J$ and $z \in C$ we have $|y-z|>\delta$. Now, if $\eta<\delta$, the curve $J_{\eta, 0}$ does not intersect with $C$, hence $x_{0}$ and $x$ are in the same side of $J_{\eta, 0}$. This verifies the claim.

Employing Claim 3 we conclude that the union of the outsides of $J_{\eta, 0}$ is Out $(J)$. This completes the proof of Proposition 4.1.

Corollary 4.2. For every $\eta>0$ and $\varepsilon>0$ there exists a twice continuously differentiable Jordan curve $J_{\eta, \varepsilon}$, each point of which is of distance between $\eta-\varepsilon$ and $\eta+\varepsilon$ from $J$, with length $L_{\eta, \varepsilon}$ less than or equal to $L+2 \pi \eta+\varepsilon$ and such that the intersection of the insides of $J_{\eta, \varepsilon}$ is $\operatorname{In}(J) \cup J$.

Proof. It is well known that a Jordan curve with a finite length can be approximated by a twice continuously differentiable Jordan curve with location and length arbitrarily close to that of the original one. (A direct proof is easy to construct based on a piecewise constant approximation in measure of the derivative, then smoothening, say a convolution, procedure. The approximation step in Tverberg [6] follows a similar argument. Alternatively, one can construct an approximation relying on the Whitney's extension theorem, see Federer [4, Theorem 3.1.16], and then apply a smoothening procedure.)

Proof of Theorem A. Consider the twice continuously differentiable curves $J_{\eta, \varepsilon}$ established in Corollary 4.2. Denote by $U_{\eta, \varepsilon}$ the set of points in 
$[0, T]$ such that $x(t)$ is in the inside of $J_{\eta, \varepsilon}$ or on $J_{\eta, \varepsilon}$. By Corollary 3.2

$$
\left|\int_{U_{\eta, \varepsilon}} u(t) d t\right| \leq \frac{L}{2}+2 \pi \eta+\varepsilon .
$$

The proof of the theorem will be concluded by taking in (4.2) the limit as $\eta$ and $\varepsilon$ go to 0 . (An alternative proof, avoiding the smooth approximation, would be to use the Auxiliary Proposition of Proposition 3.1 to show that for a set of full measure the intersection of $x(\cdot)$ with $\partial D_{\eta}$ is finite. Then use Proposition 2.2 and Claims 1 and 2 above to argue that the integral of $u(t)$ over the sets of points where $x(t)$ is in any component of the complement of $D_{\eta}$, is bounded by half the length of the boundary of that component. Now, it is easy to see that the boundaries of distinct components intersect in only a finite number of points, hence the sum of the lengths of these boundaries does not exceed $L+2 \pi \eta$. Taking now the limit as $\eta \rightarrow 0$ completes the proof.)

\section{Examples, comments}

It is clear that the consequence of Theorem A does not hold for curves in more than two dimensional space, nor for curves that are not Jordan curves. The estimate $\frac{L}{2}$ is sharp in general, but can be improved when $J$ is convex. Here are the statement and two examples.

Remark 5.1. The estimate $\frac{L}{2}$ in Theorem A can be improved in case $J$ is convex to be equal to the diameter $D$ of $J$ in $R^{2}$. Indeed, when $J$ is convex the estimate in Lemma 2.1 (that served us all along) can be set to be $D$; just follow the proof of Lemma 2.1 when $v(s)$ is replaced by the projection of $v(s)$ on a line where the diameter is attained.

Example 5.2. Let $J$ be the curve in $R^{2}$ connecting $(0,0),(1,0),(1,2 h)$ and $(0,2 h)$ and let $J_{1}$ be the curve connecting $(0, h),(1, h),(1,3 h)$ and $(0,3 h)$ (i.e., $J_{1}$ is the shift of $J$ upward by $h$ ). The value of the integral in (1.1) for this case is equal to 1 , and for $h$ small this is close to half the circumference, namely, half the length of $J$, and also close to the diameter of the convex set determined by $J$. This shows that the estimate $D$ is sharp.

Example 5.3. We show now that, in general, the estimate $\frac{L}{2}$ cannot be replaced by $D$. Consider a comb-like figure, say $C$, determined by the segment joining $(0,0)$ and $(\Delta, 0)$, and the vertical lines joining $\left(\frac{j}{N} \Delta, 0\right)$ and $\left(\frac{j}{N} \Delta, 1\right)$, for $j=0,1, \ldots, N$. Let $J$ be the boundary of all the points that are of distance $\delta$ from $C$, with $\delta$ small relative to $\frac{\Delta}{N}$. Let $J_{1}$ be the curve obtained by shifting $J$, say to the right, by $\frac{\delta}{2}$. When $\Delta$ is small and $N$ is large, then $L$ is large and the integral in (1.1) converges to $\frac{L}{2}$, while the diameter of $J$ converges to 1 .

Remark 5.4. The results of this paper extend the weaker (both the estimate and the assumptions) result employed in [1] in order to establish the existence of optimal periodic solutions to infinite horizon optimal control problem with planar state space. The reasoning in Sects. 2 and 3 follows that of [1], with the needed adaptation. However, the reasoning has been used in the proofs in [1] 
without displaying the result. Likewise with the consequence of our analysis, which we display next.

Let $J$ be a Jordan curve in $R^{2}$, generated by the absolutely continuous mapping $x(t):[0, T] \rightarrow R^{2}$. Let $u(\cdot)$ be the almost everywhere derivative of $x(\cdot)$. Along with $J$ we consider the state-velocity occupational measure, say $\boldsymbol{\mu}(J)$, induced by $x(\cdot)$ on $R^{2} \times R^{2}$, namely, the probability measure on $R^{2} \times R^{2}$ defined by

$$
\boldsymbol{\mu}(J)(E)=\frac{1}{T} \lambda(\{t \in[0, T]:(x(t), u(t)) \in E\}
$$

where $\lambda$ is the Lebesgue measure, namely, $\boldsymbol{\mu}(J)(E)$ is the proportion of time in $[0, T]$ that the trajectory $(x(\cdot), u(\cdot))$ stays in $E$ (thus, $\boldsymbol{\mu}(J)$ depends on the parameterization).

Consider now a family $J_{i}$ of Jordan curves in $R^{2}$. Let $L_{i}$ be the length of $J_{i}$. Pick a parameterization $x_{i}(\cdot)$ of $J_{i}$ and suppose that $\boldsymbol{\mu}\left(J_{i}\right)$ converges, in the weak convergence of measures, to $\boldsymbol{\mu}_{0}$ (for the terminology and results that we use in regard to probability measures see, e.g., Ash [2], Billingsley [3]). Let $\mathbf{p}$ be the marginal of $\boldsymbol{\mu}_{0}$ on the state space $R^{2}$ and let $\boldsymbol{\nu}_{0}(x)$ be the disintegration of $\boldsymbol{\mu}_{0}$ with respect to $\mathbf{p}$ (notice that for $\mathbf{p}$-almost every $x$ the measure $\boldsymbol{\nu}_{0}(x)$ is a probability measure on the $u$-variable).

Proposition 5.5. If $L_{i} \rightarrow \infty$ as $i \rightarrow \infty$ then

$$
\int_{R^{2}} u \nu_{0}(x)(d u)=0
$$

for $\mathbf{p}$-almost every $x$.

Proof. Let $x$ be in the support of $\mathbf{p}$. Consider a small ball, say $B_{x, r}$, of radius $r$ around $x$. Then, applying (1.1) for $J$ being the circumference of $B_{x, r}$ and for $x(\cdot)$ generating the Jordan curve $J_{i}$ implies that

$$
\int_{B_{x, r} \times R^{2}} u \boldsymbol{\mu}\left(J_{i}\right)(d x \times d u) \leq \frac{1}{L_{i}} 2 r .
$$

Taking the limit as $i \rightarrow \infty$ yields

$$
\int_{B_{x, r} \times R^{2}} u \boldsymbol{\mu}_{0}(d x \times d u)=0 .
$$

Since (5.4) holds for every ball, hence for every Borel set, it clearly implies (5.2). This completes the proof.

The interpretation of the previous result is the following two-dimensional phenomenon. If the density of a sequence of longer and longer Jordan curves in $R^{2}$ converges, then, in the limit, the velocities average out locally, namely, the density of velocities tends weakly to zero. Clearly, the consequence may not be true if the curves are not Jordan. 


\section{References}

[1] Artstein, Z., Bright, I.: Periodic optimization suffices for infinite horizon planar optimal control. SIAM J. Control Optim. 48, 4963-4986 (2010)

[2] Ash, R.B.: Real Analysis and Probability. Academic Press, New York (1972)

[3] Billingsley, P.: Convergence of Probability Measures, 2nd edn. Wiley, New York (1999)

[4] Federer, H.: Geometric Measure Theory. Springer, Berlin (1969)

[5] Guggenheimer, H.W.: Differential Geometry. McGraw-Hill Book Co., Inc., New York (1963)

[6] Tverberg, H.: A proof of the Jordan Curve Theorem. Bull. Lond. Math. Soc. 12, 34-38 (1980)

[7] Wells, J.C.: A note on a corollary of Sard's theorem. Proc. Am. Math. Soc 48, 513-514 (1975)

Zvi Artstein

Department of Mathematics

The Weizmann Institute of Science

Rehovot 76100

Israel

e-mail: zvi.artstein@weizmann.ac.il

Ido Bright

Department of Applied Mathematics

University of Washington

Seattle, WA 98195-2420

USA

e-mail: ibright@uw.edu

Received: 15 December 2011.

Accepted: 24 July 2012. 